\title{
Valid garnet-biotite thermometer: A comparative study
}

\author{
*H. Thomas and Haritabh Rana \\ Department of Applied Geology, School of Engineering and Technology \\ Doctor Harisingh Gour Vishwavidyalaya, Sagar (MP), India \\ *Corresponding author: harelthomas@gmail.com
}

\begin{abstract}
The abundance of garnet-biotite pair in a wide range of rocks mainly from upper green schist to granulite facies has made it one of the most widely used pairs for estimation of temperature at which once rocks equilibrated. In last four decades, more than 20 thermometer models of garnet-biotite pair have been proposed. To find the suitability of models, twenty-one thermometers formulated by a number of scholars since 1976 is considered. 27 sample data of granulites from the global literature were collected and processed through the "Gt-Bio.EXE" software. We conclude that four models are the most valid and reliable of these kinds of thermometers: Perchuk and Lavrente'va (1983); Thompson (1976); Ferry and Spear (1978) and Holdaway and Lee (1977).
\end{abstract}

Keywords: Geothermometer, Exchange reactions, Fe-Mg partitioning, Granulite, Comparative study

Paper Received: 7 Jan 2019

Paper Accepted: 20 Mar 2019

\section{INTRODUCTION}

Distribution of ferrous iron and magnesium between coexisting silicate minerals is the basis of important geothermometers for metamorphic rocks. Of wide interest is the distribution between garnet and biotite as the thermometer ranging for a wide range of rocks from greenschist to granulites. In last four decades, more than twenty thermometers have been proposed for garnet-biotite pair including both empirical and experimental calibrations. Several thermobarometric studies have been undertaken in the past few years, which led to the development of a range of thermometers such as garnet-biotite thermometer ( $\mathrm{Wu}$ and Cheng, 2006); garnet-clinopyroxene thermometer (Jahnson et al., 1983; Fu et al., 1998); garnet orthopyroxene thermometer (Thomas et al., 2018).

For last four decades, several experiments led to the development of GB thermometry. (Frost, 1962) had used the $\mathrm{Fe}-\mathrm{Mg}$ distribution between garnet and biotite to qualitatively determine the grade of metamorphic rocks. (Kretz, 1964) stated that the Fe-Mg distribution between garnet and biotite may be a function of pressure and temperature without stating any formulation.

However, the calibrations are diverse and may be confusing to petrologists in choosing a suitable version. With the development of geothermometric studies, it appears necessary to undertake a review of this thermometer every decade or so. In order to recommend the best calibration for geologists, the authors have compared twenty one garnet - biotite thermometer models proposed since 1976 applicable on granulites facies of rocks. The partitioning of the $\mathrm{Fe}^{+2}$ and $\mathrm{Mg}$, expressed by the distribution coefficient between coexisting garnet and biotite, has clearly shown that this distribution is a function of both physical conditions as well as compositional variations of the phases involved (Thompson, 1976; Holdaway and Lee, 1977; Goldman and Albee, 1977; Ferry and Spear, 1978; Pigage and Greenwood, 1982; Hodges and Spear, 1982; Perchuk and Lavrente'va, 1983; Perchuk et al., 1985; Indares and Martignole a and b, 1985; Hoinkes, a and b, 1986; Aranovich et al., 1988; Dasgupta et al., 1991; Bhattacharya et al., a and b, 1992; Perchuk, 1977, 1981; Holdaway et al., 1997; Gessman et al., 1997 and Kaneko and Miyano, a and b, 2004):

$$
\begin{aligned}
& 1 / 3 \mathrm{Fe}_{3} \mathrm{Al}_{2} \mathrm{Si}_{3} \mathrm{O}_{12}+1 / 3 \mathrm{KMg}_{3} \mathrm{AlSi}_{3} \mathrm{O} 10(\mathrm{OH})_{2} \\
& (1 / 3 \text { Almandine })+(1 / 3 \text { Phologopite }) \\
& \rightleftharpoons \begin{array}{l}
\rightleftharpoons \\
\rightleftharpoons \mathrm{Mg}_{3} \mathrm{Al}_{2} \mathrm{Si}_{3} \mathrm{O}_{12}+1 / 3 \mathrm{KFe}_{3} \mathrm{AlSi}_{3} \mathrm{O}_{10}(\mathrm{OH})_{2} \\
(1 / 3 \text { Pyrope })+(1 / 3 \text { Annite })
\end{array}
\end{aligned}
$$

The distribution of $\mathrm{Fe}$ and $\mathrm{Mg}$ between the phase's garnet and biotite is a function of pressure and temperature. As a general rule, the partitioning of elements between phases decreases as temperature increases: that is, $K_{D}$ approaches 1 and $\ln \mathrm{K}_{\mathrm{D}}$ approaches 0 .

For this the equilibrium constant, $\mathrm{K}_{\mathrm{D}}, \mathrm{T}$ at some $\mathrm{P}$ and $\mathrm{T}$ is given by:

$$
\mathrm{K}_{\mathrm{D}}, \mathrm{T}=\frac{\left(\mathrm{a}_{\mathrm{Pyr} G t}\right)^{1 / 3} *\left(\mathrm{a}_{\mathrm{Ann}} \mathrm{Bio}\right)^{1 / 3}}{\left(\mathrm{a}_{\mathrm{Alm}} \mathrm{Gt}\right)^{1 / 3} *\left(\mathrm{a}_{\mathrm{Phlo}} \mathrm{Bio}\right)^{1 / 3}}
$$

where 'a' refers to the activity of component and the superscripts refer respectively to garnet and biotite phases. If both garnet and biotite behave as ideal 3 site solid solutions 
then, taking standard states to be the pure phases at the $\mathrm{P}$ and $\mathrm{T}$ of interest, the $\mathrm{K}_{(\mathrm{P}, \mathrm{T})}$ corresponds to the empirical distribution coefficient,

$$
\mathrm{K}_{\mathrm{D}}=\left(\mathrm{X}_{\mathrm{Mg}}^{\mathrm{Gt} *} \mathrm{X}_{\mathrm{Fe}}{ }^{\mathrm{Bio}}\right) /\left(\mathrm{X}_{\mathrm{Fe}}{ }^{\mathrm{Gt} *} \mathrm{X}_{\mathrm{Mg}}^{\mathrm{Bio}}\right)
$$

Where,

$$
\begin{aligned}
& \mathrm{X}_{\mathrm{Fe}} \mathrm{GT}=\mathrm{Fe} /(\mathrm{Fe}+\mathrm{Mg}+\mathrm{Mn}+\mathrm{Ca}) ; \mathrm{X}_{\mathrm{Mg}}{ }^{\mathrm{GT}}= \\
& \mathrm{Mg} /(\mathrm{Fe}+\mathrm{Mg}+\mathrm{Mn}+\mathrm{Ca}) ; \mathrm{X}_{\mathrm{Fe}}{ }^{\mathrm{BT}}=\mathrm{Fe} /(\mathrm{Fe}+\mathrm{Mg}) ; \mathrm{X}_{\mathrm{Mg}}{ }^{\mathrm{BT}} \\
& \quad=\mathrm{Mg} /(\mathrm{Fe}+\mathrm{Mg})
\end{aligned}
$$

Several models of geothermometer have been formulated for garnet-biotite pair by a number of workers since 1976 to till dates which are summaried below:

Thompson (1976) attempted to calculate isobaric Fe-Mg section from available experimental and thermo-chemical data in the KFMASH system, calculated at $\mathrm{PH}_{2} \mathrm{O}=5 \mathrm{~kb}$ for reactions involving garnet - biotite - staurolite - chlorite - cordierite quartz - muscovite assemblages. Holdaway and Lee (1977) have given an empirical calibration of the garnet-biotite geothermometer applicable to high grade metamorphism of pelitic rocks. Ferry and Spear (1978) carried out experimental calibration with garnet-biotite ratio as $49 / 1$ and $\Delta \mathrm{H}=12,454$ $\mathrm{cal} ; \Delta \mathrm{S}=4.662 \mathrm{Cal} / \mathrm{K}$ mole to gave a polythermal polybaric equation. Perchuk $(1977,1981)$ calibrated an equation using distribution coefficient as a factor without using pressure effect in the equation. Goldman and Albee (1977) used the isotopic and chemical data for thirteen metamorphic rocks containing garnet and biotite to investigate the dependence of the $\mathrm{Mg}$-Fe partition upon temperature to give an emperical calibration. Hodges and Spear (1982) incorporated (Ferry and Spear, 1978) data set in their calibrations along with alternative calibration using a consistent set of solution models to formulate an equation. Pigage and Greenwood (1982) gave an empirical equation for coexisting garnet - biotite with sillimanite plus kyanite using $\mathrm{XCaGT}$ and $\mathrm{XMnGT}$ as variables in the equation. Perchuk and Lavrente'va (1983) undertook an experimental calibration using natural minerals and biotite with a high $\mathrm{Al}$ content. Their equation avoids the problem of non-ideality by working directly with the natural minerals. Perchuk et al. (1985) formulated an equation for calculation of temperature of metamorphism for Aldan granulites using $\mathrm{X}_{\mathrm{Ca}} \mathrm{Gt}$ as variable in the equation. Indares and Martignole (1985) proposed two new calibration of (Newton and Haselton, 1981) and (Ganguly and Saxena, 1984) based upon the correction for $\mathrm{Al}$ and $\mathrm{Ti}$ and the interaction of $\mathrm{Ca}$ in $\mathrm{Fe}$ - Mg garnets. Hoinkes (1986) gave two equations evaluating the $\mathrm{Ca}$ content in the metapelites of staurolite in zone, supporting non-ideal mixing of the grossular with almandine - pyrope solid solution. Aranovich et al. (1988) reformulated the equation using the experimental data set of Perchuk and Lavrente'va (1983). Dasgupta et al. (1991) gave a new formulation developed through statistical regression of the reversed experimental data of Ferry and Spear (1978) using available thermo-chemical data for quaternary $\mathrm{Fe}-\mathrm{Mg}-\mathrm{Ca}-\mathrm{Mn}$ garnet solid solution and for the excess free energy terms, associated with the mixing of $\mathrm{Al}$ and $\mathrm{Ti}$, in octahedral sites in biotite solid solution. Bhattacharya et al. (1992) gave two new formulations using (Ganguly and Saxena, 1984) and (Hackler and Wood, 1989) of garnet - biotite thermometer using the non-ideal mixing in the phlogopiteannite binary system. Gessman et al. (1997) used new experimental data for the Fe-Mg exchange between garnet and biotite using the Fe-Mg-Al mixing properties of biotite to give new calibration. Holdaway et al. (1997) recalibrated the equation using recently obtained Margules parameters for ternary $\mathrm{Fe}$ $\mathrm{Mg}$-Ca garnet, $\mathrm{Mn}$ interactions in garnet, $\mathrm{Al}$ interactions in biotite as well as the Fe oxidation state of both minerals. Kaneko and Miyano (2004) derived two equations, one as the presence of ferric $\mathrm{Fe}$ in biotite in relation to the coexisting $\mathrm{Fe}$-oxide phases and second assuming the absence of ferric Fe in biotite, both evaluated in terms of iterative multiple least-square regressions of the experimental results.

\section{RESULTS AND DISCUSSION}

For the validation of the software (Thomas, 1995; Thomas and Paudel, 2017) and comparative study of different models, 27 sample data of granulites (Table 1: Bohlen and Essene, 1980; Harris et al.,1982; Hodge and Spear, 1982; Keinast and Ouzegane, 1987; Perchuk, 1989; Sharma et al, 1989; Riciputi et al., 1990; Barth and May, 1992; Sen and Bhattacharya, 1992; Buchernurminen and Ohta, 1993; Liangzhao and Shiqin, 1993; Dasgupta et al., 1994; Fareduddin et al., 1994; Kumar and Chacko, 1994; Thomas,1995; Knudsen, 1996; Muhongo and Tuisku, 1996; Shaw and Arima, 1996; Bindu,1997; Ellis and Hiroi, 1997; Raith et al., 1997; Bose et al., 2001; Pattison et al., 2003; Sommer et al., 2008; Tadokaro et al., 2008; Gross et al., 2009; Yang et al., 2015) have been processed through "GtBio.EXE" software. A comparison of the calculated $\ln \mathrm{K}_{\mathrm{D}}$ and $1 / T$ for different geothermometric models has been done. The $\mathrm{K}_{\mathrm{D}}, \ln \mathrm{K}_{\mathrm{D}}, \mathrm{X}_{\mathrm{Fe}} \mathrm{Gt}, \mathrm{X}_{\mathrm{Mg}} \mathrm{Gt}, \mathrm{X}_{\mathrm{Ca}} \mathrm{Gt}, \mathrm{X}_{\mathrm{Mn}} \mathrm{Gt}, \mathrm{X}_{\mathrm{Mg}} \mathrm{Bt}, \mathrm{X}_{\mathrm{Fe}} \mathrm{Bt}$, $X_{\mathrm{Al}} \mathrm{Bt}$ and, $\mathrm{X}_{\mathrm{Ti}} \mathrm{Bt}$ of different rocks samples by different authors are shown in Table 1 and the plots of $\operatorname{lnK}_{\mathrm{D}}$ vs $1 / \mathrm{T}$ plot are shown in Figs. (1a-u) along with temperature distribution of specific models are shown in Table 2.

The data selected in this way was used to check the temperature dependence of the distribution coefficient.

Perchuk and Lavarente'va, 1983 (Fig. 1a) graph of $\ln K_{D}$ vs $1 / \mathrm{T}$ has been plotted as $\ln \mathrm{K}_{\mathrm{D}}=1875 / \mathrm{T}\left({ }^{\circ} \mathrm{C}\right)-1.470$ with $\mathrm{R}^{2}$ $=0.997$;

Thompson, 1976 (Fig. 1b) as $\ln \mathrm{K}_{\mathrm{D}}=1567 / \mathrm{T}\left({ }^{\circ} \mathrm{C}\right)-$ 0.425 with $\mathrm{R}^{2}=0.996$;

Ferry and Spear, 1978 (Fig. 1c) as $\ln K_{\mathrm{D}}=1020 / \mathrm{T}\left({ }^{\circ} \mathrm{C}\right)$ -0.040 with $\mathrm{R}^{2}=0.992$;

Holdaway and Lee, 1977 (Fig. 1d) as $\operatorname{lnK}_{\mathrm{D}}=1402 / \mathrm{T}$ $\left({ }^{\circ} \mathrm{C}\right)-0.723$ with $\mathrm{R}^{2}=0.963$;

Hoinkes, 1986(b) (Fig. 1e) as $\ln \mathrm{K}_{\mathrm{D}}=1032 / \mathrm{T}\left({ }^{\circ} \mathrm{C}\right)-$ 0.003 with $\mathrm{R}^{2}=0.951$;

Perchuk, 1977; 1981 (Fig. 1f) as $\ln K_{\mathrm{D}}=1271 / \mathrm{T}\left({ }^{\circ} \mathrm{C}\right)-$ 0.591 with $\mathrm{R}^{2}=0.926$; 
Hoinkes, 1986 A, (Fig. 1g) as $\ln K_{\mathrm{D}}=1038 / \mathrm{T}\left({ }^{\circ} \mathrm{C}\right)+$ 0.063 with $\mathrm{R}^{2}=0.921$;

Kaneko and Miyano, 2004(b) (Fig. 1h) as $\operatorname{lnK}_{\mathrm{D}}=1570 /$ $\mathrm{T}\left({ }^{\circ} \mathrm{C}\right)-1.07$ with $\mathrm{R}^{2}=0.909$;

Indares and Martingole, (a. Newton and Haselton, 1981), 1985 (Fig. 1i) as $\ln \mathrm{K}_{\mathrm{D}}=882.6 / \mathrm{T}\left({ }^{\circ} \mathrm{C}\right)-0.070$ with $\mathrm{R}^{2}=0.903$;

Perchuk et al., 1985 (Fig. $1 \mathrm{j})$ as $\ln \mathrm{K}_{\mathrm{D}}=1740 / \mathrm{T}\left({ }^{\circ} \mathrm{C}\right)-$ 1.234 with $\mathrm{R}^{2}=0.871$;

Hodges and Spear, 1982 (Fig. $1 \mathrm{k}$ ) as $\ln \mathrm{K}_{\mathrm{D}}=1044 / \mathrm{T}$ $\left({ }^{\circ} \mathrm{C}\right)-0.048$ with $\mathrm{R}^{2}=0.870$;

Holdaway et al., 1997 (Fig. 11) as $\ln \mathrm{K}_{\mathrm{D}}=-759.1 / \mathrm{T}\left({ }^{\circ} \mathrm{C}\right)$ +2.607 with $\mathrm{R}^{2}=0.855$;

Pigage and Greenwood, 1982 (Fig. $1 \mathrm{~m}$ ) as $\ln \mathrm{K}_{\mathrm{D}}=1210 /$ $\mathrm{T}\left({ }^{\circ} \mathrm{C}\right)-0.158$ with $\mathrm{R}^{2}=0.851$; Aranovich et al., 1988 (Fig. 1n) as $\operatorname{lnKD}=1264 / \mathrm{T}\left({ }^{\circ} \mathrm{C}\right)-0.332$ with $\mathrm{R}^{2}=0.780$;

Bhattacharya et al., 1992 (a) (Fig. 1o) as $\operatorname{lnK}_{\mathrm{D}}=985 / \mathrm{T}$ $\left({ }^{\circ} \mathrm{C}\right)-0.089$ with $\mathrm{R}^{2}=.767$;

Indares and Martingole, (b. Ganguly and Saxena, 1984), 1985 (Fig. 1p) as $\ln \mathrm{K}_{\mathrm{D}}=837.3 / \mathrm{T}\left({ }^{\circ} \mathrm{C}\right)+0.028$ with $\mathrm{R}^{2}=0.674$;

Dasgupta et al., 1991 (Fig. 1q) as $\ln \mathrm{K}_{\mathrm{D}}=523.8 / \mathrm{T}\left({ }^{\circ} \mathrm{C}\right)$
+0.729 with $\mathrm{R}^{2}=0.659$

Kaneko and Miyano, 2004 (a) (Fig. 1r) as $\ln K_{D}=1364$ / $\mathrm{T}\left({ }^{\circ} \mathrm{C}\right)-0.655$ with $\mathrm{R}^{2}=0.652$; Goldman and Albee, 1977 (Fig. $1 \mathrm{~s})$ as $\ln \mathrm{KD}=747.5 / \mathrm{T}\left({ }^{\circ} \mathrm{C}\right)+0.240$ with $\mathrm{R}^{2}=0.647$;

Gessman et al., 1997 (Fig. $1 \mathrm{t}$ ) as $\operatorname{lnK}_{\mathrm{D}}=844.9 / \mathrm{T}\left({ }^{\circ} \mathrm{C}\right)$ + 0.236 with $\mathrm{R}^{2}=0.599$; and Bhattacharya et al., 1992 (b) (Fig. $1 \mathrm{u})$ as $\ln \mathrm{K}_{\mathrm{D}}=402.3 / \mathrm{T}\left({ }^{\circ} \mathrm{C}\right)+0.819$ with $\mathrm{R}^{2}=0.526$.

On the basis of different plots, it is observed that Perchuk and Lavrente'va (1983); Thompson (1976); Ferry and Spear (1978) and Holdaway and Lee (1977) are showing very good relation between $\operatorname{lnKD}$ vs $1 / \mathrm{T}$ and maximum points are coming in best fit lines and has high regression values.

\section{CONCLUSION}

Among the twenty-one geothermometer models of garnetbiotite equilibrium considered for this comparative study, Perchuk and Lavrente'va (1983); Thompson (1976); Ferry and Spear (1978) and Holdaway and Lee (1977) are showing the highest regression values and maximum points (values of temperature) are coming in best fit lines (Figs. 1a-d). So, these models can be considered empirically as the most appropriate ones to be used for the calculation of temperature.

Table 1: Data of $K_{D}$, $\ln K_{D}, X_{F e} G t, X_{M g} G t, X_{C a} G t, X_{M n} G t, X_{M g} B t, X_{F e} B t, X_{A l}$ Bt and, $X_{T i}$ Bt of different rocks samples by different authors

\begin{tabular}{|c|c|c|c|c|c|c|c|c|c|c|}
\hline Data of different authors & KD & LNKD & $\mathrm{X}_{\mathrm{Fe}}(\mathrm{GT})$ & $\mathrm{X}_{\mathrm{Mg}}(\mathrm{GT})$ & $\mathrm{X}_{\mathrm{Mn}}(\mathrm{GT})$ & $\mathrm{X}_{\mathrm{Ca}}(\mathrm{GT})$ & $\mathrm{X}_{\mathrm{Fe}}(\mathrm{BT})$ & $\mathrm{X}_{\mathrm{Mg}}(\mathrm{BT})$ & $\mathrm{X}_{\mathrm{Ti}}(\mathrm{BT})$ & $\mathrm{X}_{\mathrm{Al}}(\mathrm{BT})$ \\
\hline 1. Fareduddin et al., 1994 & 6.5854 & 1.8849 & 0.683162 & 0.151777 & 0.021754 & 0.143308 & 0.405997 & 0.594003 & 0.085745 & 0.029296 \\
\hline 2. Sharma et al., 1989 & 4.4993 & 1.5039 & 0.581613 & 0.193871 & 0.012581 & 0.211935 & 0.400040 & 0.599960 & 0.100088 & 0.020720 \\
\hline 3. Sen and Bhattacharya, 1992 & 4.0973 & 1.4103 & 0.613666 & 0.350065 & 0.010687 & 0.025583 & 0.299643 & 0.700357 & 0.056345 & 0.070562 \\
\hline 4. Perchuk, 1989 & 6.2770 & 1.8369 & 0.760623 & 0.18748 & 0.023678 & 0.028219 & 0.392593 & 0.607407 & 0.017389 & 0.371606 \\
\hline 5. Bose et al., 2001 & 11.4956 & 2.442 & 0.566195 & 0.014509 & 0.349655 & 0.069641 & 0.772458 & 0.227542 & 0.063475 & 0.123322 \\
\hline 6. Harris et al., 1982 & 3.9128 & 1.3642 & 0.585634 & 0.325906 & 0.058863 & 0.029598 & 0.314717 & 0.682830 & 0.107725 & 0.023033 \\
\hline 7. Muhongo and Tuisku, 1996 & 3.6956 & 1.3071 & 0.517309 & 0.295791 & 0.018040 & 0.168861 & 0.321225 & 0.678775 & 0.089482 & 0.022152 \\
\hline 8. Barth and May, 1992 & 3.3232 & 1.2009 & 0.575908 & 0.214851 & 0.028713 & 0.180528 & 0.446472 & 0.553528 & 0.125373 & 0.006848 \\
\hline 9. Riciputi et al., 1990 & 2.7304 & 1.0045 & 0.570910 & 0.364404 & 0.031746 & 0.032759 & 0.364666 & 0.635334 & 0.056667 & 0.149000 \\
\hline 10. Liangzhao and Shiqin, 1993 & 3.2373 & 1.1747 & 0.601329 & 0.335548 & 0.006645 & 0.056478 & 0.356322 & 0.643678 & 0.073333 & 0.056667 \\
\hline 11. Kumar and Chacko,1994 & 3.0268 & 1.1075 & 0.717260 & 0.240430 & 0.023842 & 0.018469 & 0.496975 & 0.503625 & 0.100652 & 0.049602 \\
\hline 12. Keinast and Ouzegane, 1987 & 4.1082 & 1.413 & 0.542448 & 0.444255 & 0.007842 & 0.005455 & 0.229120 & 0.770880 & 0.103177 & 0.013924 \\
\hline 13. Dasgupta et al., 1994 & 4.9774 & 1.6049 & 0.535802 & 0.371289 & 0.018163 & 0.074747 & 0.224763 & 0.775237 & 0.070607 & 0.011233 \\
\hline 14. Tadokaro et al., 2008 & 4.9868 & 1.6068 & 0.636270 & 0.335276 & 0.005828 & 0.022626 & 0.275654 & 0.724346 & 0.087427 & 0.058166 \\
\hline 15. Buchernurminen and Ohta, 1993 & 4.9351 & 1.5964 & 0.795918 & 0.159864 & 0.017007 & 0.027211 & 0.502203 & 0.497797 & 0.086806 & 0.012500 \\
\hline 16. Gross et al., 2009 & 3.3293 & 1.2028 & 0.700997 & 0.255814 & 0.132890 & 0.029900 & 0.451477 & 0.548523 & 0.100000 & 0.535710 \\
\hline 17. Shaw and Arima, 1996 & 6.9161 & 1.9339 & 0.681116 & 0.184140 & 0.026882 & 0.107863 & 0.348459 & 0.654541 & 0.069143 & 0.021051 \\
\hline 18. Yang et al., 2015 & 6.8372 & 1.9224 & 0.653333 & 0.286667 & 0.033330 & 0.026667 & 0.250000 & 0.750000 & 0.043860 & 0.114035 \\
\hline 19. Raith et al., 1997 & 3.7479 & 1.3212 & 0.520392 & 0.420881 & 0.016313 & 0.042414 & 0.248062 & 0.751938 & 0.072414 & 0.037931 \\
\hline 20. Knudsen, 1996 & 4.5030 & 1.5047 & 0.676768 & 0.292929 & 0.010101 & 0.020202 & 0.339093 & 0.660907 & 0.098039 & 0.076649 \\
\hline 21. Ellis and Hiroi, 1997 & 2.7227 & 1.0016 & 0.668901 & 0.253016 & 0.043566 & 0.034517 & 0.492641 & 0.507359 & 0.105982 & 0.022476 \\
\hline 22. Bindu, 1997 & 3.9599 & 1.3762 & 0.708169 & 0.264493 & 0.010211 & 0.017128 & 0.403394 & 0.596606 & 0.123702 & 0.035890 \\
\hline 23. Bohlen and Essene, 1980 & 3.6507 & 1.2949 & 0.698552 & 0.132705 & 0.109801 & 0.058942 & 0.590480 & 0.409520 & 0.061833 & 0.152667 \\
\hline 24. Thomas, 1995 & 6.9285 & 1.9356 & 0.726952 & 0.233728 & 0.013454 & 0.025867 & 0.309824 & 0.690176 & 0.080045 & 0.065651 \\
\hline 25. Sommer et al., 2008 & 4.2565 & 1.4485 & 0.622074 & 0.274247 & 0.030100 & 0.073579 & 0.347640 & 0.652360 & 0.038732 & 0.137324 \\
\hline 26. Pattison et al., 2003 & 3.1028 & 1.1323 & 0.516667 & 0.380000 & 0.066670 & 0.036667 & 0.304688 & 0.695313 & 0.083624 & 0.020906 \\
\hline 27. Hodge and Spear, 1982 & 7.4259 & 2.0050 & 0.730000 & 0.100000 & 0.140000 & 0.030000 & 0.495726 & 0.504273 & 0.025729 & 0.168096 \\
\hline
\end{tabular}


Table 2: Data of the Calculated Temperature $\left({ }^{\circ} \mathrm{C}\right)$ of different rocks samples by different authors

\begin{tabular}{|c|c|c|c|c|c|c|c|c|c|c|c|}
\hline Data of different authors & $\begin{array}{l}\text { Thompson } \\
\text { (1976) }\end{array}$ & $\begin{array}{l}\text { Holdway } \\
\text { and Lee } \\
\text { (1977) }\end{array}$ & $\begin{array}{l}\text { Goldman } \\
\text { and Albee } \\
\text { (1977) }\end{array}$ & $\begin{array}{l}\text { Ferry } \\
\text { and } \\
\text { Spear } \\
(1978)\end{array}$ & $\begin{array}{l}\text { Pigage and } \\
\text { Greenwood } \\
(1982)\end{array}$ & $\begin{array}{l}\text { Hodges } \\
\text { and } \\
\text { Spear } \\
(1982)\end{array}$ & $\begin{array}{l}\text { Perchuk and } \\
\text { Laverante'va } \\
\text { (1983) }\end{array}$ & $\begin{array}{l}\text { Perchuk } \\
\text { et al. } \\
\text { (1985) }\end{array}$ & $\begin{array}{l}\text { Indares and } \\
\text { Martingole } \\
\text { (1985a) }\end{array}$ & $\begin{array}{l}\text { Indares and } \\
\text { Martingole } \\
\text { (1985b) }\end{array}$ & $\begin{array}{l}\text { Hoinkes } \\
\text { (1986a) }\end{array}$ \\
\hline 1. Fareduddin et al., 1994 & 682 & 551 & 497 & 535 & 631 & 590 & 560 & 608 & 482 & 499 & 640 \\
\hline 2. Sharma et al., 1989 & 819 & 641 & 647 & 670 & 824 & 752 & 633 & 717 & 611 & 647 & 850 \\
\hline 3. Sen and Bhattacharya, 1992 & 858 & 666 & 629 & 710 & 735 & 721 & 653 & 641 & 627 & 613 & 744 \\
\hline 4. Perchuk, 1989 & 697 & 561 & 622 & 550 & 579 & 561 & 569 & 560 & 507 & 442 & 576 \\
\hline 5. Bose et al., 2001 & 534 & 447 & 379 & 396 & 572 & 420 & 473 & 484 & 346 & 488 & 435 \\
\hline 6. Harris et al., 1982 & 879 & 679 & 618 & 732 & 789 & 744 & 663 & 653 & 572 & 597 & 772 \\
\hline 7. Muhongo and Tuisku, 1996 & 906 & 695 & 756 & 759 & 898 & 826 & 676 & 740 & 686 & 725 & 956 \\
\hline 8. Barth and May, 1992 & 959 & 728 & 710 & 814 & 977 & 886 & 701 & 772 & 683 & 734 & 1043 \\
\hline 9. Riciputi et al.,1990 & 1071 & 794 & 888 & 934 & 986 & 947 & 750 & 741 & 822 & 803 & 999 \\
\hline 10. Liangzhao and Shiqin, 1993 & 973 & 736 & 704 & 829 & 879 & 852 & 707 & 712 & 721 & 714 & 920 \\
\hline 11. Kumar and Chacko,1994 & 1010 & 758 & 612 & 868 & 900 & 876 & 723 & 707 & 690 & 681 & 901 \\
\hline 12. Keinast and Ouzegane, 1987 & 857 & 665 & 599 & 709 & 718 & 711 & 652 & 630 & 550 & 537 & 716 \\
\hline 13. Dasgupta et al., 1994 & 779 & 615 & 600 & 630 & 690 & 660 & 613 & 627 & 561 & 574 & 708 \\
\hline 14. Tadokaro et al., 2008 & 778 & 614 & 556 & 629 & 648 & 639 & 612 & 600 & 509 & 491 & 654 \\
\hline 15. Buchernurminen and Ohta, 1993 & 782 & 617 & 469 & 633 & 661 & 644 & 614 & 604 & 509 & 482 & 664 \\
\hline 16. Gross et al., 2009 & 958 & 727 & 1384 & 813 & 925 & 826 & 700 & 690 & 650 & 635 & 861 \\
\hline 17. Shaw and Arima, 1996 & 667 & 540 & 489 & 521 & 597 & 562 & 552 & 582 & 476 & 494 & 602 \\
\hline 18. Yang et al., 2015 & 670 & 543 & 526 & 524 & 556 & 534 & 554 & 544 & 470 & 463 & 547 \\
\hline 19. Raith et al., 1997 & 899 & 691 & 685 & 752 & 794 & 770 & 673 & 670 & 650 & 651 & 812 \\
\hline 20. Knudsen, 1996 & 818 & 640 & 565 & 670 & 689 & 678 & 633 & 619 & 525 & 502 & 694 \\
\hline 21. Ellis and Hiroi, 1997 & 1073 & 795 & 658 & 936 & 998 & 730 & 751 & 743 & 748 & 769 & 1004 \\
\hline 22. Bindu, 1997 & 874 & 675 & 549 & 726 & 745 & 733 & 660 & 644 & 535 & 517 & 749 \\
\hline 23. Bohlen and Essene, 1980 & 912 & 699 & 639 & 765 & 879 & 789 & 679 & 685 & 674 & 717 & 849 \\
\hline 24. Thomas, 1995 & 666 & 540 & 472 & 520 & 542 & 530 & 552 & 542 & 425 & 412 & 542 \\
\hline 25. Sommer et al., 2008 & 842 & 655 & 688 & 694 & 763 & 723 & 645 & 659 & 652 & 649 & 782 \\
\hline 26. Pattison et al., 2003 & 996 & 750 & 744 & 853 & 929 & 868 & 717 & 711 & 719 & 762 & 916 \\
\hline 27. Hodge and Spear, 1982 & 645 & 526 & 482 & 501 & 583 & 512 & 540 & 532 & 467 & 510 & 524 \\
\hline
\end{tabular}

Table 2: Continued.

\begin{tabular}{|c|c|c|c|c|c|c|c|c|c|c|}
\hline Data of different authors & $\begin{array}{l}\text { Hoinkes } \\
\text { (1986b) }\end{array}$ & $\begin{array}{l}\text { Aranovich } \\
\text { et al. } \\
\text { (1988) }\end{array}$ & $\begin{array}{l}\text { Dasgupta } \\
\text { et al. } \\
\text { (1991) }\end{array}$ & $\begin{array}{l}\text { Bhattacharya } \\
\text { et al. } \\
\text { (1992a) }\end{array}$ & $\begin{array}{l}\text { Bhattacharya } \\
\text { et al. } \\
\text { (1992b) }\end{array}$ & $\begin{array}{l}\text { Perchuk, } \\
(1977, \\
1981)\end{array}$ & $\begin{array}{l}\text { Holdaway } \\
\text { et al. } \\
\text { (1997) }\end{array}$ & $\begin{array}{l}\text { Gessman } \\
\text { et al. } \\
1997\end{array}$ & $\begin{array}{l}\text { Kaneko and } \\
\text { Miyano } \\
(2004 a)\end{array}$ & $\begin{array}{l}\text { Kaneko and } \\
\text { Miyano } \\
(2004 b)\end{array}$ \\
\hline 1. Fareduddin et al., 1994 & 597 & 659 & 635 & 588 & 603 & 537 & 1006 & 532 & 563 & 547 \\
\hline $\begin{array}{l}\text { 2. Sharma et al., } 1989 \\
\text { 3. Sen and Bhattacharya, } 1992\end{array}$ & $\begin{array}{l}788 \\
718\end{array}$ & $\begin{array}{l}770 \\
712\end{array}$ & $\begin{array}{l}812 \\
815\end{array}$ & $\begin{array}{l}679 \\
642\end{array}$ & $\begin{array}{l}697 \\
638\end{array}$ & $\begin{array}{l}620 \\
643\end{array}$ & $\begin{array}{l}715 \\
684\end{array}$ & $\begin{array}{l}636 \\
683\end{array}$ & $\begin{array}{l}655 \\
670\end{array}$ & $\begin{array}{l}637 \\
641\end{array}$ \\
\hline 4. Perchuk, 1989 & 550 & 496 & 437 & 575 & 591 & 546 & 978 & 820 & 583 & 548 \\
\hline 5. Bose et al., 2001 & 414 & 465 & 274 & 343 & 217 & 384 & 1153 & 530 & 452 & 438 \\
\hline 6. Harris et al., 1982 & 743 & 753 & 813 & 656 & 661 & 635 & 659 & 661 & 674 & 653 \\
\hline $\begin{array}{l}\text { 7. Muhongo and Tuisku, } 1996 \\
\text { 8. Barth and May, } 1992\end{array}$ & $\begin{array}{l}865 \\
929\end{array}$ & $\begin{array}{l}815 \\
840\end{array}$ & $\begin{array}{l}937 \\
909\end{array}$ & $\begin{array}{l}713 \\
741\end{array}$ & $\begin{array}{l}736 \\
759\end{array}$ & $\begin{array}{l}665 \\
690\end{array}$ & $\begin{array}{l}642 \\
534\end{array}$ & $\begin{array}{l}685 \\
759\end{array}$ & $\begin{array}{l}716 \\
722\end{array}$ & $\begin{array}{l}693 \\
704\end{array}$ \\
\hline $\begin{array}{l}\text { 9. Riciputi et al.,1990 } \\
\text { 10. Liangzhao and Shiqin, } 1993\end{array}$ & $\begin{array}{l}952 \\
860\end{array}$ & $\begin{array}{l}816 \\
805\end{array}$ & $\begin{array}{l}937 \\
920\end{array}$ & $\begin{array}{l}749 \\
711\end{array}$ & $\begin{array}{l}753 \\
713\end{array}$ & $\begin{array}{l}744 \\
707\end{array}$ & $\begin{array}{l}472 \\
545\end{array}$ & $\begin{array}{l}985 \\
790\end{array}$ & $\begin{array}{l}744 \\
732\end{array}$ & $\begin{array}{l}706 \\
702\end{array}$ \\
\hline 11. Kumar and Chacko,1994 & 865 & 795 & 828 & 726 & 728 & 720 & 468 & 874 & 721 & 697 \\
\hline $\begin{array}{l}\text { 12. Keinast and Ouzegane,1987 } \\
\text { 13. Dasgupta et al., } 1994\end{array}$ & $\begin{array}{l}710 \\
669\end{array}$ & $\begin{array}{l}747 \\
728\end{array}$ & $\begin{array}{l}841 \\
818\end{array}$ & $\begin{array}{l}623 \\
610\end{array}$ & $\begin{array}{l}610 \\
615\end{array}$ & $\begin{array}{l}643 \\
594\end{array}$ & $\begin{array}{l}713 \\
851\end{array}$ & $\begin{array}{l}601 \\
537\end{array}$ & $\begin{array}{l}663 \\
639\end{array}$ & $\begin{array}{l}643 \\
618\end{array}$ \\
\hline 14. Tadokaro et al., 2008 & 634 & 675 & 721 & 601 & 598 & 599 & 818 & 596 & 616 & 595 \\
\hline 15. Buchernurminen and Ohta, 1993 & 631 & 651 & 571 & 620 & 632 & 599 & 741 & 637 & 604 & 589 \\
\hline 16. Gross et al., 2009 & 819 & 777 & 808 & 704 & 706 & 697 & 525 & 1313 & 641 & 605 \\
\hline 17. Shaw and Arima, 1996 & 564 & 646 & 645 & 573 & 591 & 526 & 1090 & 497 & 553 & 537 \\
\hline 18. Yang et al., 2015 & 529 & 580 & 616 & 548 & 555 & 526 & 1110 & 544 & 564 & 538 \\
\hline 19. Raith et al., 1997 & 777 & 780 & 907 & 660 & 661 & 662 & 665 & 666 & 703 & 676 \\
\hline $\begin{array}{l}\text { 20. Knudsen, } 1996 \\
\text { 21. Ellis and Hiroi, } 1997\end{array}$ & $\begin{array}{l}671 \\
947\end{array}$ & $\begin{array}{l}680 \\
834\end{array}$ & $\begin{array}{l}706 \\
919\end{array}$ & $\begin{array}{l}628 \\
757\end{array}$ & $\begin{array}{l}628 \\
761\end{array}$ & $\begin{array}{l}621 \\
743\end{array}$ & $\begin{array}{l}728 \\
426\end{array}$ & $\begin{array}{l}669 \\
897\end{array}$ & $\begin{array}{l}630 \\
768\end{array}$ & $\begin{array}{l}607 \\
744\end{array}$ \\
\hline $\begin{array}{l}\text { 22. Bindu, } 1997 \\
\text { 23. Bohlen and Essene, } 1980 \\
\text { 24. Thomas, } 1995\end{array}$ & $\begin{array}{l}723 \\
786 \\
521\end{array}$ & $\begin{array}{l}727 \\
666 \\
598\end{array}$ & $\begin{array}{l}735 \\
651 \\
590\end{array}$ & $\begin{array}{l}659 \\
669 \\
555\end{array}$ & $\begin{array}{l}661 \\
658 \\
569\end{array}$ & $\begin{array}{l}653 \\
644 \\
531\end{array}$ & $\begin{array}{l}630 \\
515 \\
1107\end{array}$ & $\begin{array}{l}702 \\
915 \\
522\end{array}$ & $\begin{array}{l}652 \\
667 \\
551\end{array}$ & $\begin{array}{l}633 \\
637 \\
532\end{array}$ \\
\hline 25. Sommer et al., 2008 & 729 & 716 & 760 & 654 & 663 & 630 & 697 & 749 & 658 & 626 \\
\hline $\begin{array}{l}\text { 26. Pattison et al., } 2003 \\
\text { 27. Hodge and Spear, } 1982\end{array}$ & $\begin{array}{l}875 \\
500\end{array}$ & $\begin{array}{l}830 \\
514\end{array}$ & $\begin{array}{l}976 \\
463\end{array}$ & $\begin{array}{l}714 \\
518\end{array}$ & $\begin{array}{l}728 \\
513\end{array}$ & $\begin{array}{l}691 \\
491 \\
\end{array}$ & $\begin{array}{l}557 \\
1068 \\
\end{array}$ & $\begin{array}{l}751 \\
630 \\
\end{array}$ & $\begin{array}{l}760 \\
542\end{array}$ & $\begin{array}{l}733 \\
516\end{array}$ \\
\hline
\end{tabular}

However, Perchuk and Lavrente'va (1983) is the best among them as the regression correlation coefficient value; $\mathrm{R}^{2}$ is close to 1 which indicates that the maximum points are coming in best fit line. Therefore, the temperature value obtained by the Perchuk and Lavrente'va (1983) model is more accurate as compared to others. 


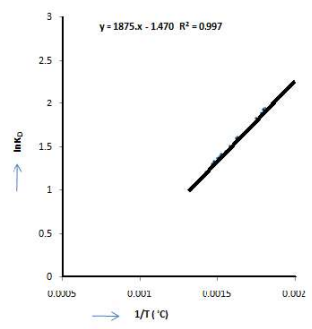

(a)

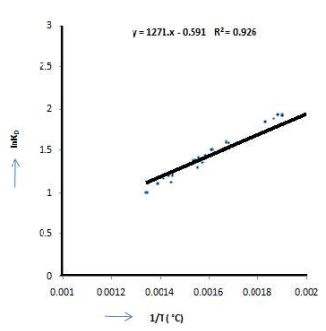

(f)

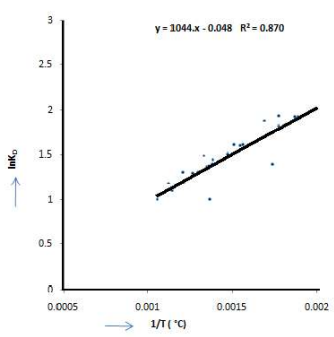

(k)

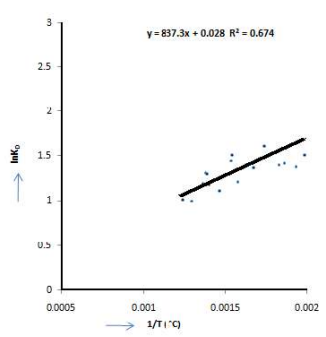

(p)

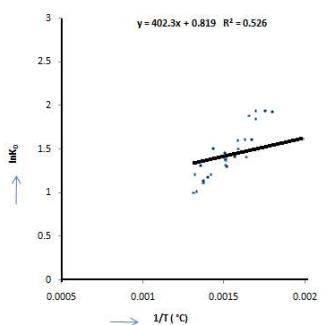

(u)

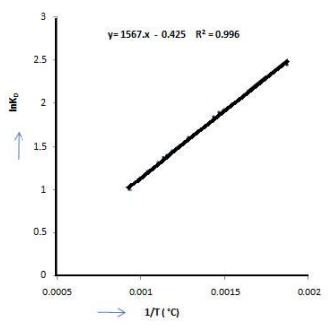

(b)

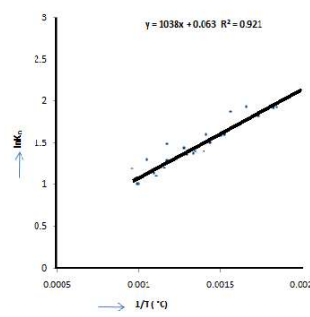

(g)

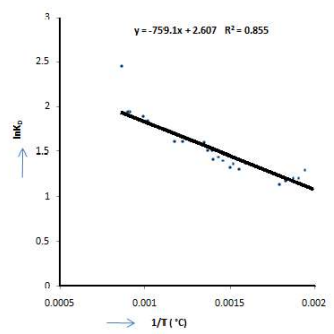

(I)

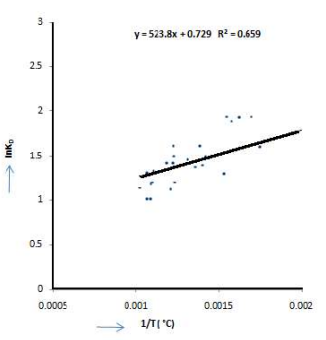

(q)

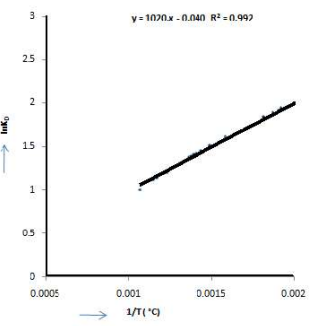

(c)

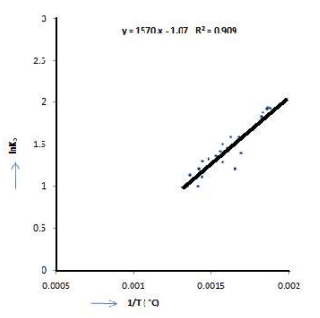

(h)

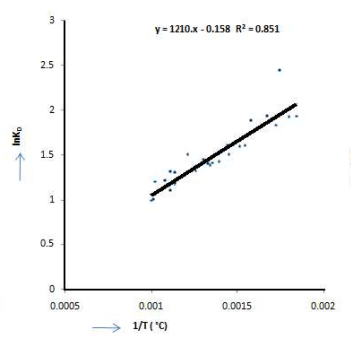

(m)

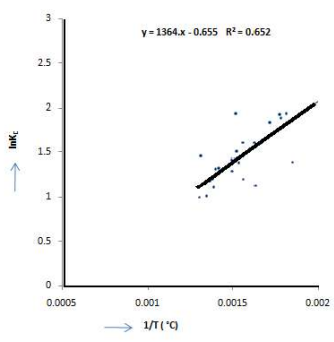

(r)

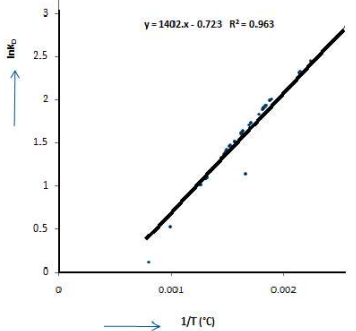

(d)

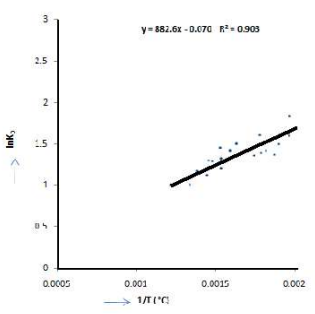

(i)

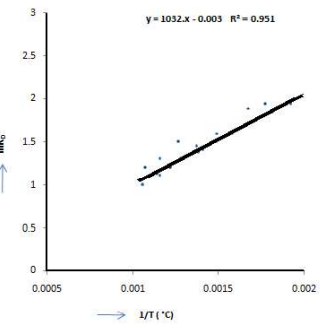

(e)

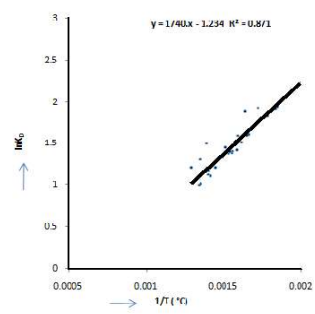

(j)

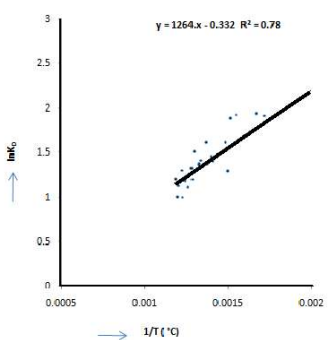

(n)

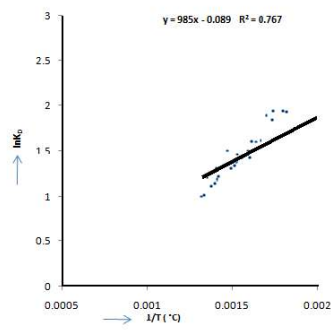

(o)

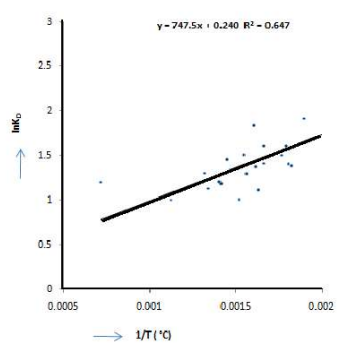

(s)

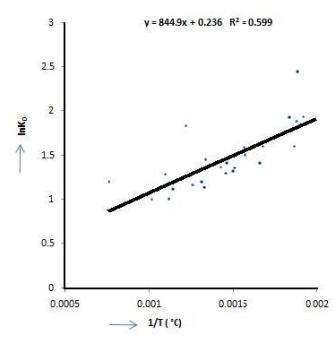

(t)

Fig. 1: (a) Perchuk and Lavarente'va, 1983, (b) Thompson, 1976, (c) Ferry and Spear, 1978, (d) Holdaway and Lee, 1977, (e) Hoinkes, 1986(b), (f) Perchuk, 1977; 1981, (g) Hoinkes, 1986 a, (h) Kaneko and Miyano, 2004(b), (i) Indares and Martingole, (a. Newton and Haselton, 1981), 1985, (j) Perchuk et al., 1985, (k) Hodges and Spear, 1982, (l) Holdaway et al., 1997, (m) Pigage and Greenwood, 1982, (n) Aranovich et al., 1988, (o) Bhattacharya et al., 1992, (p) Indares and Martingole, (b. Ganguly and Saxena, 1984), 1985, (q) Dasgupta et al., 1991, (r) Kaneko and Miyano, 2004 (a), (s) Goldman and Albee, 1977, (t) Gessman et al., 1997, and (u) Bhattacharya et al., 1992 (b) 


\section{ACKNOWLEDGEMENT}

The authors thank the Head, Department of Applied Geology, Doctor Harisingh Gour Vishwavidyalaya, Sagar (M.P.) and the Department of Science and Technology, New Delhi, India for providing facilities as including PURSE- Phase -II for conducting present research work. The authors express sincere thanks to the reviewer, Prof., University of Torino, Italy, for his critical and constructive reviews which significantly improved the focus and clarity of the manuscript.

\section{REFERENCES}

Aranovich, L., Ya, I., Lavrent'eva, V., and Kosyakova, N.A., 1988, Biotite-garnet and biotite orthopyroxene geothermometers: calibrations accounting for the $\mathrm{Al}$ variations in biotite. Geokhimiya, v. 5, pp. 668-676.

Barth, A.P. and May, D.J., 1992, Mineralogy and pressuretemperature-time path of cretaceous granulite gneisses, south-Eastern San Gabriel Mountains, Southern California. Journal of Metamorphic Geology, v. 10(4), pp. 529-544.

https://doi.org/10.1111/j.1525-1314.1992.tb00103.x

Bhattacharya, A., Mohanty, L., Maji, A., Sen, S.K., and Raith, M., 1992, Non-ideal mixing in the phlogopite-annite binary: constraints from experimental data on $\mathrm{Mg}-\mathrm{Fe}$ partitioning and a reformulation of the biotite-garnet geothermometer. Contribution to Minerology and Petrology, v. 111(1), pp. 87-93.

https://doi.org/10.1007/BF00296580

Bindu, R.S., 1997, Granulite facies spinel-cordierite assemblages from the Kerala khondalite belt, southern India. Gondwana Research, v. 1, pp. 121-128.

https://doi.org/10.1016/S1342-937X(05)70010-1

Bohlen, S.R. and Essene, E.J., 1980, Evaluation of coexisting garnet-biotite, garnet- clinopyroxene, and other mg-fe exchange thermometers in adirondack granulites. Geological Society of American Bulletin, v. 91(2), pp. 685-719.

https://doi.org/10.1130/GS AB-P2-91-685

Bose, S., Fukuoka, M., Sengupta, P., and Dasgupta, S., 2000, Evolution of high- Mg-Al granulites from Sunkarametta, Eastern Ghats, India: Evidence for a lower crustal heatingcooling trajectory. Journal of Metamorphic Geology, v. 18(3), pp. 223-240.

https://doi.org/10.1046/j.1525-1314.2000.00253.x

Buchernurminen, K. and Ohta, Y., 1993, Granulites and garnetcordierite gneisses from Dronning Maud Land, Antarctica. Journal of Metamorphic Geology, v. 11(5), pp. 691-703.

https://doi.org/10.1111/j.1525-1314.1993.tb00181.x

Dasgupta, S., Sengupta, P., Guha, D., and Fukuoka, M., 1991, A refined garnet-biotite Fe-Mg exchange geothermometer and its application in amphibolites and granulites.
Contribution to Minerology and Petrology, v. 109(1), pp. 130-137.

https://doi.org/10.1007/BF00687206

Dasgupta, S., Sanyal, S., Sengupta, P., and Fukuoka, M., 1994, Petrology of granulites from Anakapalle - evidence for proterozoic decompression in the eastern ghats, India. Journal of Petrology, v. 35(2), pp. 433-459.

https://doi.org/10.1093/petrology/35.2.433

Ellis, D.J. and Hiroi, Y., 1997, Secondary siderite - oxide sulphide and carbonate- andalusite assemblages in cordierite granulites from Sri Lanka: post-granulite facies fluid evolution during uplift. Contribution to Minerology and Petrology, v. 127(4), pp. 315-335.

https://doi.org/10.1007/s004100050283

Fareeduddin, S.M., Basavalingu, B., and Janardhan, A.S., 1994, P-T conditions of pelitic granulites and associated charnockites of Chinwali area, west of Delhi fold belt, Rajasthan. Journal of Geological Society, India, v. 43(2), pp. 169-178.

Ferry, J.M.T. and Spear, F.S., 1978, Experimental calibration of the partitioning of $\mathrm{Fe}$ and $\mathrm{Mg}$ between biotite and garnet. Contribution to Minerology and Petrology, v. 66(2), pp. 113-117.

https://doi.org/10.1007/BF00372150

Frost, M.J., 1962, Metamorphic grade and iron-magnesium distribution between co-existing garnet-biotite and garnethornblende. Geological Magazine, v. 99(5), pp. 427-438.

https://doi.org/10.1017/S0016756800059690

Fu, B., Zheng, Y.F., Li, Y.L., and Li, S.G., 1998, Applications of garnet-clinopyroxene geothermometers to eclogite assemblages. Acta Mineralogica Sinica, v. 18, pp. 145-157.

Ganguly, J. and Saxena, S.K., 1984, Mixing properties of alumina silicate garnets: Constraints from natural and experimental data, and applications to geothermobarometry. American Mineralogist, v. 69(1-2), pp. 88-97.

Gessmann, C.K., Spiering, B., and Raith, M., 1997, Experimental study of the Fe-Mg exchange between garnet and biotite: Constraints on the mixing behaviour and analysis of the cation-exchange mechanisms. American Mineralogist, v. 82(11-12), pp. 1225-1240.

https://doi.org/10.2138/am-1997-11-1218

Goldman, D.S. and Albee, A.L., 1977, Correlation of Mg/Fe partitioning between garnet and biotite with/Sup 18/O//Sup 16/O partitioning between quartz and magnetite. American Journal of Science, (6), 277p.

https://doi.org/10.2475/ajs.277.6.750

Gross, A. O. M. S., Droop, G. T. R., Porcher, C. C., and Fernandes, L. A. D., 2009, Petrology and thermobarometry of mafic granulites and migmatites from the Chafalote metamorphic suite: New Insights into the neoproterozoic P-T evolution 
of the uruguayan - sul - rio - Grandense Shield. Precambrian Research, v.170(3), pp. 157-174.

https://doi.org/10.1016/j.precamres.2009.01.011

Hackler, R. and Wood, B., 1989, Experimental-determination of $\mathrm{Fe}$ and $\mathrm{Mg}$ exchange between garnet and olivine and estimation of $\mathrm{Fe}-\mathrm{Mg}$ mixing properties in garnet. American Mineralogist, v. 74(9-10), pp. 994-999.

Harris, N.B.W., Holt, R.W., and Drury, S.A., 1982, Geobarometry, geothermometry, and Late Archean geotherms from the granulite facies Terrain of south India. Journal of Geology, v. 90(5), pp. 509-527. https://doi.org/10.1086/628709

Hodges, K.V. and F.S. Spear., 1982, Geothermometry, geobarometry and the $\mathrm{Al}_{2} \mathrm{SiO}_{5}$ triple point at Mt Moolsanke, New Hamphire. American Mineralogist, v. 67, pp. 1118-1134.

Hoinkes, G., 1986, Effect of grossular - content in garnet on the partitioning of $\mathrm{Fe}$ and $\mathrm{Mg}$ between garnet and biotite. Contribution to Minerology and Petrology, v. 92(3), pp. 393-399.

\section{https://doi.org/10.1007/BF00572168}

Holdaway, M.J. and Lee, S.M., 1977, Fe-Mg cordierite stability in high-grade pelitic rocks based on experimental, theoretical, and natural observation. Contribution to Minerology and Petrology, v. 63, pp. 175-198.

https://doi.org/10.1007/BF00398778

Holdaway, M.J., Mukhopadhyay, B., Dyar, M.D., Guidotti, C.V., and Dutrow, B.L., 1997, Garnet - Biotite geothermometry revised: New margules parameters and a natural specimen data set from maine. American Mineralogist, v. 82(5-6), pp. 582-595.

https://doi.org/10.2138/am-1997-5-618

Indares, A. and Martignole J., 1985, Biotite - garnet geothermometry in granulite facies: The influence of Ti and $\mathrm{Al}$ in biotite. American Mineralogist, v. 70, pp. 272278.

Jahnson, C.A., Bohlen, S.R., and Essene, E.J., 1983, An evaluation of garnet-clinopyroxene geothermometry in granulites. Contribution to Minerology and Petrology, v. 84, pp. 191-198.

https://doi.org/10.1007/BF00371285

Kaneko, Y. and Miyano, T., 2004, Recalibration of mutually consistent garnet-biotite and garnet-cordierite geothermometers. Lithos, v. 73(3), pp. 255-269.

https://doi.org/10.1016/j.lithos.2003.12.009

Kienast, J.R. and Ouzegane, K., 1987, Polymetamorphic Al, Mg-rich granulites with orthopyroxene - sillimanite and sapphirine parageneses in Archaean rocks from the Hoggar, Algeria. Geological Journal, v. 22(S2), pp. 57-79. https://doi.org/10.1002/gj.3350220607
Knudsen, T.L., 1996, Petrology and geothermobarometry of granulite facies metapelites from the Hisøy-Torungen area, South Norway: New data on the Sveconorvegian PT-t path of the Bamble sector. Journal of Metamorphic Geology, v. 14(3), pp. 267-287.

https://doi.org/10.1111/j.1525-1314.1996.00267.x

Kretz, R., 1964, Analysis of equilibrium in garnet-biotitesillimanite gneisses from Quebec. Journal of Petrology, v. 5(1), pp. 1-20.

https://doi.org/10.1093/petrology/5.1.1

Kumar, C.R. and Chacko, T., 1994, Geothermobarometry of mafic granulites and metapelite from the palghat gap, south India: Petrological evidence for isothermal uplift and rapid cooling. Journal of Metamorphic Geology, v. 12(4), pp. 479-492.

https://doi.org/10.1111/j.1525-1314.1994.tb00037.x

Liangzhao, L. and Shiqin, J., 1993, P-T-t Paths and tectonic history of an Early Precambrian granulite facies terrane, Jining District, South-East Inner Mongolia, China. Journal of Metamorphic Geology, v. 11(4), pp. 483-498.

https://doi.org/10.1111/j.1525-1314.1993.tb00166.x

Muhongo, S. and Tuisku, P., 1996, Pan-African high pressure isobaric cooling: evidence from the mineralogy and thermobarometry of the granulite-facies rocks from the Uluguru mountains, eastern Tanzania. Journal of African Earth Sciences, v. 23(3), pp. 443-463.

https://doi.org/10.1016/S0899-5362(97)00012-2

Newton, R.C. and Haselton, H.T., 1981, Thermodynamics of the garnet-plagioclase-A12SiO5-quartz geobarometer. In:ÊThermodynamics of minerals and melts: $\hat{E}$ New York, (Springer), pp. 131-147,

https://doi.org/10.1007/978-1-4612-5871-1_7

Pattison, D.R., Chacko, T., Farquhar, J., and Mcfarlane, C.R., 2003, Temperatures of granulite - facies metamorphism: constraints from experimental phase equilibria and thermobarometry corrected for retrograde exchange. Journal of Petrology, v. 44(5), pp. 867-900.

https://doi.org/10.1093/petrology/44.5.867

Perchuk, L.L., 1977, Thermodynamic control of metamorphic processes: energetics of geological processes., Berlin Heidelberg (Springer), pp. 285-352.

https://doi.org/10.1007/978-3-642-86574-9_14

Perchuk, L.L., 1981, Correction of biotite-garnet thermometer for the case of $\mathrm{Mn}$ reversible $\mathrm{Mg}+\mathrm{Fe}$ isomorphism in garnet. Doklady akademii nauk sssr, v. 256(2), pp. 441442.

Perchuk, L.L. and Lavrente'va, I.V., 1983, Experimental investigation of exchange equilibria in the system cordierite - garnet - biotite. Kinetics and equilibrium in mineral reactions. Advances in Physical Geochemistry, v. 3, pp. 
199-239.

https://doi.org/10.1007/978-1-4612-5587-1_7

Perchuk, L.L., Aranovich, L.Y., Podlesskii, K.K., Lavrente'va, I.V., Gerasimov, V.Y., Fed'kin, V.V., and Berdnikov, N.V., 1985, Precambrian granulites of the Aldan shield, eastern Siberia, USSR. Journal of Metamorphic Geology, v. 3(3), pp. 265-310.

https://doi.org/10.1111/j.1525-1314.1985.tb00321.x

Perchuk, L.L., 1989, PT-fluid regimes of metamorphism and related magmatism with specific reference to the granulitefacies Sharyzhalgay complex of Lake Baikal. Geological Society of London, Special Publication, v. 43(1), pp. 275-291.

https://doi.org/10.1144/GSL.SP.1989.043.01.19

Pigage, L.C. and Greenwood, H.J., 1982, Internally consistent estimates of pressure and temperature; the staurolite problem.American Journal of Science, v. 282(7), pp. 943-969.

https://doi.org/10.2475/ajs.282.7.943

Raith, M., Karmakar, S., and Brown, M., 1997, Ultra-hightemperature metamorphism amd Multistage decompressional evolution of sapphirine granulites from the Palni hill ranges, southern India. Journal of Metamorphic Geology, v. 15(3), pp. 379-399.

https://doi.org/10.1111/j.1525-1314.1997.00027.x

Riciputi, L.R., Valley, J.W., and Mcgregor, V.R., 1990, Conditions of archaean granulite metamorphism in the GodthabFiskenaesset region, Southern west greenland. Journal of Metamorphic Geology, v. 8(2), pp. 171-190.

https://doi.org/10.1111/j.1525-1314.1990.tb00464.x

Sen, S.K. and Bhattacharya, A., 1987, Granulites of satnuru and madras: a study in different behaviour of fluids. granulites and crustal evolution. Springer Netherlands. pp. 367-384.

https://doi.org/10.1007/978-94-009-2055-2_18

Sharma, R.S., Jane D.S., and Joshi, M., 1989, Mineralogy and metamorphic history of norite dykes within granulite facies gneisses from Sand Mata, Rajasthan, NW India. Mineralogical Magazine, v. 51, pp. 207-215.

https://doi.org/10.1180/minmag.1987.051.360.03

Shaw, R.K. and Arima, M., 1996, Mineral chemistry, reaction textures, thermobarometry and P-T path from orthopyroxene granulites of Rayagada, eastern Ghats, India. Journal of Southeast Asian Earth Sciences, v. 14(34), pp. 175-184.

https://doi.org/10.1016/S0743-9547(96)00056-6

Sommer, H., Hauzenberger, C., Kröner, A., and Muhongo, S.,
2008, Isothermal decompression history in the western granulite terrain, central Tanzania: evidence from reaction textures and trapped fluids in metapelites. Journal of African Earth Science, v. 51(3), pp. 123-144.

https://doi.org/10.1016/j.jafrearsci.2008.01.003

Tadokoro, H., Toshiaki, T., and Santosh, M., 2008, Metamorphic P-T Path of the eastern Trivandrum granulite block, southern India: implications for regional correlation of lower crustal fragments. Journal of Minerological Petrological Science, v. 103(4), pp. 279-284.

https://doi.org/10.2465/jmps.080110

Thomas, H., 1995, MPET1: A computer program for coexisting garnet-biotite-aluminosilicate - plagioclase - quartz geothermobarometry. Journal of Himalayan Geology, v. 6(1), pp. 69-74.

Thomas, H., 1995, Pressure Temperature considerations for granulite from Thana-Gyangarh, district-Bhilwara, Rajasthan: implication for crustal evolution. in: R.K. Shrivastava and R. Chandra (Eds.), Magmatism in Relation to diverse tectonic setting. Oxford. IBH Pub. com. Pvt. Ltd. pp. 439-456.

Thomas, H. and Paudel, L., 2017, Garnet-biotite pair: a suitable geothermometer. Journal of Nepal Geological Society, v. 53, pp. 57-62.

https://doi.org/10.3126/jngs.v53i0.23806

Thomas, H., Rana, H., and Shahid, M. 2018, Garnetorthopyroxene (GOPX) geothermometer: a comparative study. Arabian Journal of Geosciences, v. 11(24), pp. 771-778.

https://doi.org/10.1007/s12517-018-4060-y

Thompson, A.B., 1976, Mineral reactions in pelitic rocks; Ii, calculation of some P-T- X (Fe-Mg) phase relations. American Journal of Science, v. 276(4), pp. 425-454.

https://doi.org/10.2475/ajs.276.4.425

Wu, C.M. and Cheng, B.H., 2006, Valid garnet - biotite (GB) geothermometry and garnet-aluminum silicate-plagioclasequartz (GASP) geobarometry in metapelitic rocks. Lithos, v. 89 , pp. 1-23.

https://doi.org/10.1016/j.lithos.2005.09.002

Yang, X., Li, Z., Wang, H., Chen, H., Li, Y., and Xiao, W., 2015, Petrology and geochemistry of ultra high-temperature granulites from the South Altay orogenic belt, northwestern China: implications for metamorphic evolution and protolith composition. Island Arc., v. 24(2), pp. 169-187.

https://doi.org/10.1111/iar.12102 